\title{
CIÊNCIANATURA
}

\section{Aplicação da Transformada de Hilbert-Huang em dados de velocidade medidos em túnel de vento}

\author{
Application of the Hilbert-Huang Transform in velocity data measured in wind tunnel
}

Luís Gustavo Nogueira Martins, Giuliano Demarco, Franciano Scremin Puhales, Gervásio Annes Degrazia e Otávio Costa Acevedo

Universidade Federal de Santa Maria, RS, Brasil

\section{Resumo}

Neste estudo, a transformada de Hilbert-Huang foi aplicada à medidas experimentais realizadas em túnel de vento para determinação da frequência de ocorrência do máximo espectral. Esta frequências de máximo estão associadas à escala temporal dos turbilhões mais energético e representa um importante parâmetro de entrada nos modelos de dispersão de poluentes. Os valores da frequências de máximo obtidos através do espectro marginal de Hilbert-Huang são comparados com os valores obtidos pela transformada de Fourier, tradicionalmente utilizada. Os resultados mostram que a distribuição de energia calculadas por ambos os métodos são muito semelhantes na região de ocorrência do máximo espectral, e por esse motivo, os valores de frequência de máximo obtidas por cada método não apresentam diferenças significativas. Sob certa hipótese, a transformada de Hilbert-Huang forneceu valores de frequência de máximo fisicamente mais realistas daqueles obtidos usando a transformada de Fourier.

Palavras-chave: Túnel de vento, Turbulência, Espectro de Hilbert-Huang.

\section{Abstract}

In this study, the Hilbert-Huang transform was applied to experimental measurements performed in a wind tunnel to determine the frequency of occurrence of the spectral maximum. These maximum frequencies are associated with the time scale of the most energetic eddies and represent an important input parameter in the pollutants dispersion models. The values of the maximum frequencies obtained by the Hilbert-Huang marginal spectrum are compared with the vaues obtained by the traditionally used Fourier transform. The results show that the energy distribution calculated by both methods are very similar in the region of occurence of the spectral maximum, and for this reason, the maximum frequency values obtained by each method do not present significant differences. Under determined hypothesis, the Hilbert-Huang transform provided physically more realistic maximum frequency values that those obtained using the Fourier transform.

Keywords: Wind Tunnel, Turbulence, Hilbert-Huang Transform. 


\section{Introdução}

Os espectros de energia turbulenta são ferramentas estocásticas importantes na determinação de parâmetros turbulentos em diferentes tipos de camada limite. A partir dos espectros de velocidade turbulenta pode-se determinar grandezas como as variâncias das velocidades, as frequências associadas aos máximos espectrais e, desta forma, estimar as escalas e os comprimentos característicos que descrevem as estruturas turbulentas em diferentes tipos de escoamentos Degrazia et al. (2000); Wittwer et al. (2016).

Estas informações resultantes dos espectros turbulentos permitem determinar quantidades como os coeficientes de difusão turbulentos e as escalas de tempo locais de descorrelação. Estes parâmetros alimentam os modelos de dispersão Eulerianos e Lagrangianos que permitem estimar o transporte de escalares e sua concentração em diferentes regiões do campo de escoamento.

A frequência de ocorrência do máximo espectral $\left(f_{\max }\right)$ representa a escala temporal dos turbilhões mais energético e, por esse motivo, é um parâmetro fundamental na parametrização das propriedades do transporte realizado pela turbulência nos modelos de dispersão de poluentes. Tradicionalmente, este parâmetro é obtido através da representação espectral de Fourier. No presente estudo, a transformada de Hilbert-Huang é aplicada à um conjunto de medidas de velocidade realizadas em um túnel de vento. Valores de $f_{\max }$ observados nos espectros marginais de Hilbert-Huang são comparados com aqueles obtidos pelo método clássico de Fourier.

\section{Transformada Hilbert-Huang}

A transformada de Hilbert $(y(t))$ de um sinal $x(t)$ é definida pela convolução:

$$
y(t)=\frac{1}{\pi} P \int_{-\infty}^{\infty} \frac{x(\tau)}{t-\tau} d \tau,
$$

onde $P$ representa o valor principal de Cauchy. Após a obtenção do par de transformada $x(t)$ e $y(t)$, o sinal analítico pode ser escrito como:

$$
\begin{gathered}
z(t)=x(t)+i y(t)=a(t) e^{i \theta(t)}, \text { onde } i=\sqrt{-1}, \\
a(t)=\left[x(t)^{2}+y(t)^{2}\right]^{\frac{1}{2}} \text { e } \theta(t)=\arctan \left(\frac{y(t)}{x(t)}\right) .
\end{gathered}
$$

Na Eq. 3, $a(t)$ é a amplitude e $\theta(t)$ é a função de fase.

Logo, a frequência instantânea pode ser definida como:

$$
f(t)=\frac{1}{2 \pi} \frac{d \theta(t)}{d t} .
$$

A frequência instantânea, definida pela Eq. 4, somente apresenta sentido físico quando a transformada de Hilbert é aplicada à um conjunto específico de funções, chamadas de Funções de Modo Intrínsecos (Intrinsic Mode Function - IMF). Essas funções são caracterizadas por apresentar as seguintes propriedades: a) o número de máximos ou mínimos locais é igual ao número de vezes que a função cruza o zero, ou diferente de uma unidade; b) em qualquer instante de tempo, a média local do envelope formado pela interpolação do extremos (máximos e mínimos) é igual a zero.

A primeira etapa da HHT é decompor a série temporal em um conjunto de IMFs $\left(C_{i}\right)$ utilizando um algorítimo iterativo de "peneiramento"chamado Decomposição em Modos Empíricos (DME)Huang et al. (1998). Esse algoritmo permite obter o conjunto de IMFs de uma série temporal $(x(t))$ através dos seguintes passos: 1) identificar os máximos e mínimos locais; 2) obter o envelope que confina o sinal através da interpolação dos máximos/mínimos locais; 3$)$ determinar o valor médio $\left(m_{1}(t)\right)$ desse envelope; 4) obter o primeiro protomodo: $h_{1}=x(t)-m_{1}(t)$. Caso $h_{1}$ apresente as propriedades de uma IMF, este protomodo é identificado como a primeira IMF $\left(C_{1}\right)$, que representa os modos naturais de oscilação de mais alta frequência do sinal. 5) Subtraímos $C_{1}$ do sinal original para obtermos o primeiro resíduo $r_{1}\left(r_{1}=x(t)-C_{1}\right)$. Repetimos os passos $1-5$ em $r_{1}$, e nos subsequentes resíduos, para a obtenção das demais IMFs $\left(C_{i}\right)$ de menores frequências. Este processo é repetido até que nenhuma IMF possa ser obtida do sinal residual. Neste caso, $r_{n}$ é uma função monotônica ou apresenta apenas um máximo local. Com isso, uma série temporal pode ser representada por

$$
x(t)=\sum_{i=1}^{n} C_{i}+r_{n} .
$$

Em geral, os protomodos $\left(h_{k}\right)$, obtidos no passo 4 da descrição do processo da EMD, não apresentam as propriedades de uma IMF na primeira iteração. Por esse motivo, o processo de peneiramento (passos 1-4) é aplicado repetidamente nos protomodos $h_{k, j}, j=1, \ldots, n$ até que um critério de parada seja atingido Huang et al. (1999, 2003). 


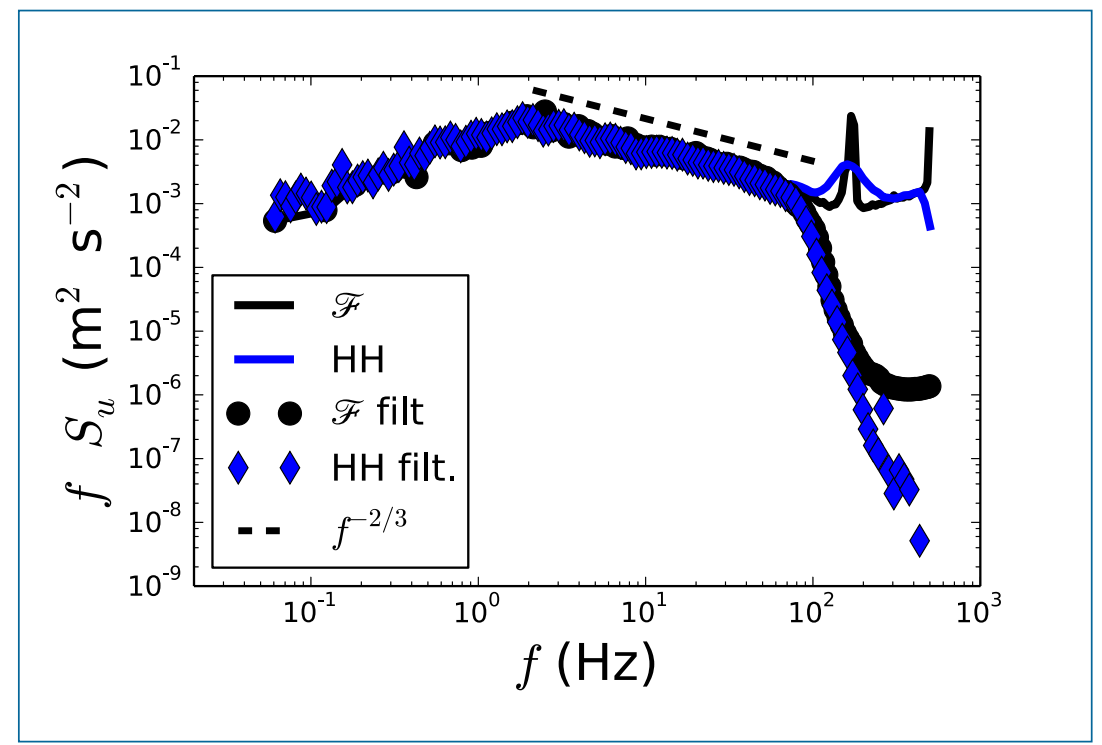

Figura 1: Espectros de Fourier $(\mathscr{F})$ e Hilbert-Huang $(\mathrm{HH})$ calculados para uma série de medidas de velocidade realizadas no nível de $50 \mathrm{~mm}$ do ensaio conduzido com $u_{\infty}=1 \mathrm{~m} \mathrm{~s}^{-1}$ (linhas contínuas). Espectros calculados para as séries filtradas (círculos e losangos).

Depois de obtido o conjunto de IMFs $\left(C_{i}\right.$, com $\left.i=1, . ., n\right)$, a transformada de Hilbert é aplicada em cada uma das IMFs e uma função analítica (equação 2) é obtida para cada caso. Desta maneira, uma série de entrada $x(t)$ pode ser representada na forma:

$$
x(t)=\sum_{i=1}^{n} a_{j}(t) \exp ^{\frac{i}{2 \pi} \int f(t) d t}
$$

A equação 6 fornece a amplitude e a frequência de cada componente como função do tempo. Adicionalmente, esta representação possibilita obter a amplitude e a frequência instantânea como funções do tempo em um diagrama tridimensional na qual a amplitude fica evidenciada no plano tempo/frequência. Este diagrama tridimensional é chamado de Espectro de Hilbert-Huang $(H(f, t))$. A integral no tempo deste espectro é chamado de espectro marginal de Hilbert-Huang $(S(f))$. Isto permite a comparação da distribuição de energia obtida pela HHT com outras representações espectrais, como a de Fourier.

\section{Descrição da obtenção dos dados}

Os dados utilizados para esta análise foram obtidos de experimentos realizados em túnel de vento aerodinâmico subsônico (TA-3), situado na Divisão de Aerodinâmica (ALA) do Instituto de Aeronáutica e Espaço (IAE) pertencente ao Departamento de Ciência e Tecnologia Aeroespacial (DCTA). O túnel opera em circuito fechado, velocidade máxima na seção em torno de $35 \mathrm{~m} / \mathrm{s}$ e intensidade turbulenta de aproximadamente $2 \%$, com uma seção de ensaios fechada, do tipo quadrada, com dimensões de 500 mm de altura, $720 \mathrm{~mm}$ de largura e $1200 \mathrm{~mm}$ de comprimento.

Para se obter o efeito de um gradiente vertical de temperatura, uma placa de alumínio foi colocada sobre o piso da seção e resfriada com água e gelo, colocados dentro de uma caixa que se encontrava em contato sob a placa. A temperatura mínima obtida sobre a placa foi de aproximadamente $5{ }^{\circ} \mathrm{C}$. Para o controle de temperatura da placa utilizou-se um multímetro digital FLUKE SÉRIE 170.

O controle de velocidade do vento gerado no túnel foi realizado com tubo de Pitot Airflow (NPL Type) conectado a um manômetro GE Sensing modelo Druck DPI 610. Para os processos de aquisição de dados, utilizou-se a anemometria de fio-quente, com uma frequência de amostragem de $1 \mathrm{kHz}$ e um tempo de aquisição de dois minutos.

Foram executados ensaios com velocidades de escoamento livre $\left(u_{\infty}\right)$ variando de 1 a $5 \mathrm{~m} \mathrm{~s}^{-1}$ em 13 nível verticais $(1,2,3,4$, $5,10,15,20,30,50,100,150$ e $250 \mathrm{~mm})$.

\section{Resultados}

Utilizando este mesmo conjunto de medidas, Puhales et al. (2015) identificou anomalias nas funções estruturas calculadas devido à presença de ruído de alta frequência. Neste mesmo estudo, foi utilizado um processo de filtragem (passa-baixa) baseado na DME 


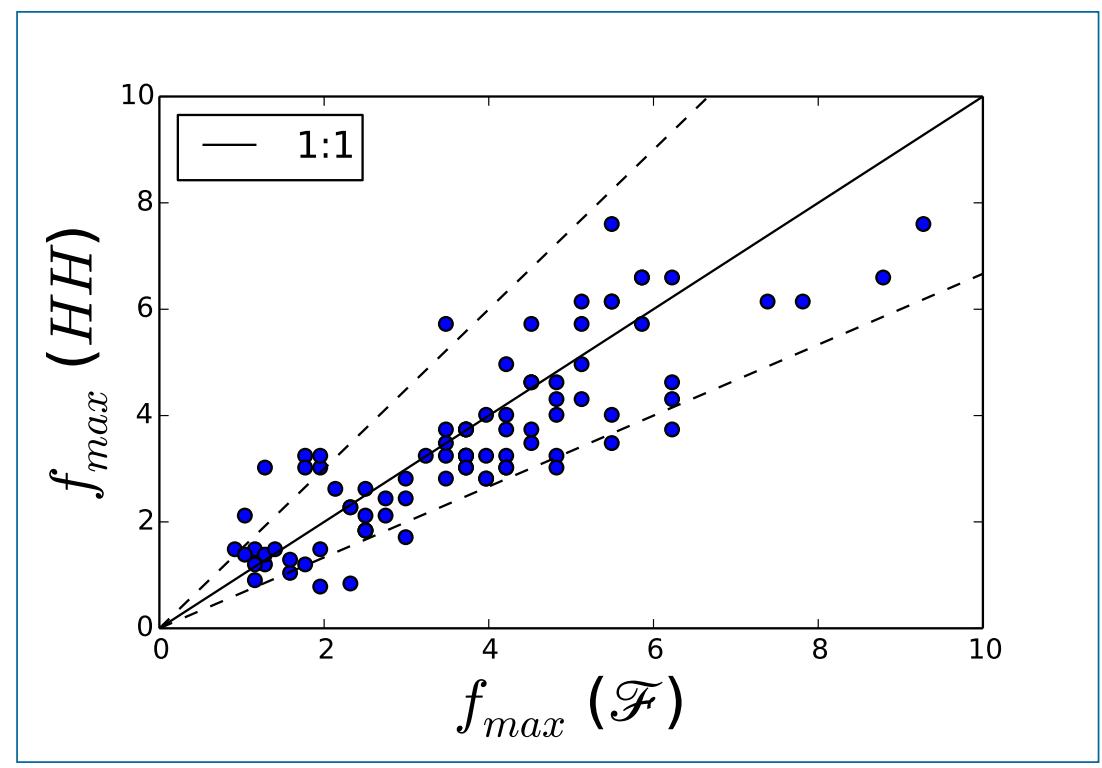

Figura 2: Gráfico de espalhamento das frequências dos máximos espectrais $\left(f_{\max }\right)$ obtidas pelas transformadas de Fourier e Hilbert-Huang.

juntamente com uma análise das funções de densidade de probabilidade para determinar o comprimento das séries temporais no qual medidas robustas das estatística de segunda ordem são obtidas. Seguindo esta mesma metodologia, para cada série de medidas de 131 segundos a DME é aplicada e as componentes ruidosas são removidas quando a série é reconstruída excluindo-se as duas primeiras IMFs de mais alta frequência. Depois disto, os espectros de Hilbert-Huang e Fourier são determinados para cada seguimento de $2^{14}$ pontos das séries de medidas. Neste análise, os diferentes níveis verticais do escoamento no túnel, em cada ensaio, é caracterizado por um espectro médio calculado a partir dos espectros de cada um dos seguimentos.

Na Figura 1, são apresentados os espectros de Fourier $(\mathscr{F})$ e Hilbert-Huang $(\mathrm{HH})$ calculados para as séries de medidas de velocidade realizadas no nível de $50 \mathrm{~mm}$ do ensaio conduzido com $u_{\infty}=1 \mathrm{~m} \mathrm{~s}^{-1}$. Pode-se observar claramente que os picos espectrais na alta frequência, associados à erros experimentais, comprometem a determinação de $f_{\text {max }}$ nos espectros das séries não filtradas, principalmente na representação de Fourier. No entanto, esta limitação é completamente eliminada pelo processo de filtragem. Embora a informação espectral de alta frequência $(f \geq 100 \mathrm{~Hz})$ tenha sido comprometida pelo processo de filtragem, o intervalo de frequências associado ao subintervalo inercial e ao máximo espectral não são afetados por este processo. Por outro lado, o espectro de Hilbert-Huang mostrou-se mais sensível ao processo de filtragem de alta frequência, além de ter uma melhor resolução na região das baixas frequências.

Outro resultado importante, que pode ser notado na Figura 1, é que a distribuições de energia calculadas pela transforama de Fourier e de Hilbert-Huang concordam em boa parte do intervalo de frequências, especialmente na região do máximo espectral. Na Figura 2, é apresentado o gráfico de espalhamento das $f_{\max }$ obtidas pelas transformadas de Fourier e Hilbert-Huang. Os valores de $f_{\max }$ obtidos em ambas as representações espectrais não apresentam diferenças significativas. Este resultado suporta a hipótese sugerida neste trabalho, de que o espectro de Hilbert-Huang pode ser utilizado em substituição ao espectro de Fourier para determinar $f_{\max }$, e consequentemente, os coeficientes de difusão turbulenta usados em modelos de dispersão.

Estimativas de $f_{\max }$ estão fortemente sujeitos à incertezas decorrente do método de decomposição escolhido e de incertezas arbitrárias resultante da metodologia utilizada. Por este motivo, é muito difícil definir qual metodologia fornece valores fisicamente mais realísticos de $f_{\max }$.

Em escoamentos em túneis de vento com grandes valores de Reynolds, é razoável assumir homogeneidade espacial das propriedades estatísticas da turbulência, assim como o valor de $f_{\max }$. Os perfis verticais de $f_{\max }$ obtidos pelas transformadas de Hilbert-Huang e Fourier são apresentados na Figura 3. Para esta análise foi utilizado as medidas do ensaio com maior valor de $V_{\infty}$ $\left(5 \mathrm{~m} \mathrm{~s}^{-1}\right)$. Esta análise mostra que os valores de $f_{\max }$ obtidos nos diferentes níveis verticais pela transformada de Hilbert-Huang apresenta uma menor variação $\left(\sigma_{f} \approx 0.5\right)$ do que a transformada de Fourier $\left(\sigma_{f} \approx 1.4\right)$. 


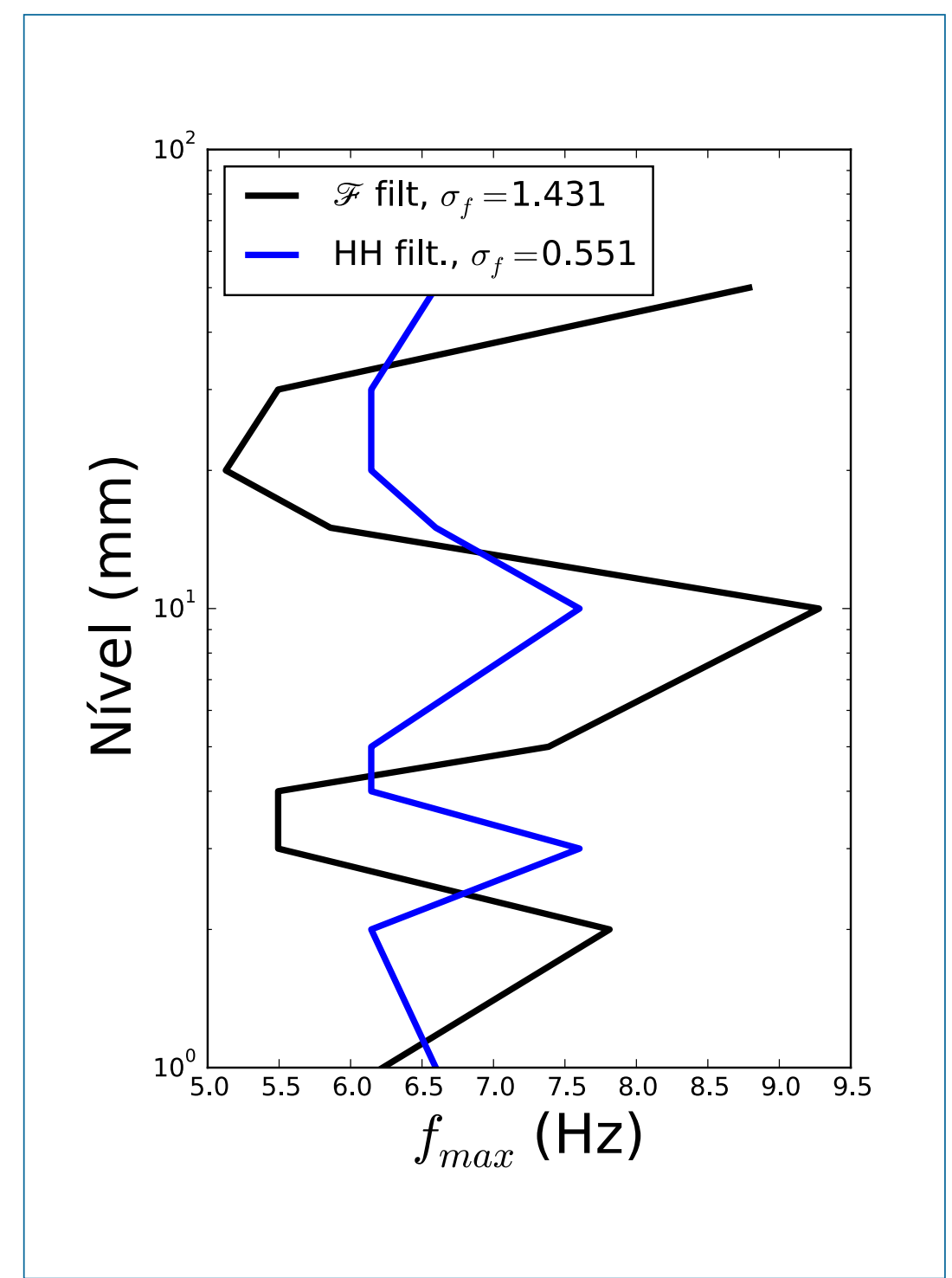

Figura 3: Perfil vertical de $f_{\max }$ observados nos espectros de Fourier e de Hilbert-Huang calculados a partir das medidas realizadas no ensaio com $u_{\infty}=5 \mathrm{~m} \mathrm{~s}^{-1}$.

\section{Conclusão}

Os coeficientes de difusão turbulenta são parâmetros de entrada fundamentais em modelos de dispersão (Lagrangianos e Eulerianos) de poluentes. Algumas parametrizações desses coeficientes dependem da frequência de ocorrência do máximo espectral, obtidas tradicionalmente pelo transformada de Fourier. Neste trabalho, dados experimentais de velocidade medidos em túnel de vento são utilizados para validar a substituição da representação espectral de Fourier pelo espectro marginal de Hilbert-Huang na determinação das frequências de máximo. Os resultados obtidos mostram que as distribuições de energia obtida por ambas abordagens concordam muito bem no intervalo de frequência do subintervalo inercial e do máximo espectral.

A análise do espalhamento dos valores de $f_{\max }$ mostra que os resultados obtidos para ambos os métodos não apresentam diferenças significativas. No entanto, uma análise mais detalhada deve ser realizada. Por exemplo, avaliar o efeito dessas diferenças nas estimativas dos coeficientes de difusão turbulenta.

Por fim, assumindo a hipótese de homogeneidade espacial em condição de turbulência completamente desenvolvida, pode-se observar que a transformada de Hilbert-Huang obteve valores fisicamente mais realistas que a transformada de Fourier. 


\section{Agradecimentos}

Os autores agradecem a Coordenação de Aperfeiçoamento de Pessoal de Nível Superior (Capes) e o Programa de Pós-graduação em Meteorologia (PPGMET-UFSM) pelo apoio proporcionado no desenvolvimento do trabalho.

\section{Referências}

Degrazia, G., Anfossi, D., Carvalho, J., Mangia, C., Tirabassi, T., Velho, H. C. (2000). Turbulence parameterisation for pbl dispersion models in all stability conditions. Atmospheric environment, 34(21), 3575-3583.

Huang, N. E., Shen, Z., Long, S. R., Wu, M. C., Shih, H. H., Zheng, Q., Yen, N. C., Tung, C. C., Liu, H. H. (1998). The empirical mode decomposition and the Hilbert spectrum for nonlinear and non-stationary time series analysis. P Roy Soc Lond A Mat, 454(1971), 903-995.

Huang, N. E., Shen, Z., Long, S. R. (1999). A new view of nonlinear water waves: The hilbert spectrum. Annu Rev Fluid Mech 31(1), 417-457.

Huang, N. E., Wu, M. L. C., Long, S. R., Shen, S. S., Qu, W., Gloersen, P., Fan, K. L. (2003). A confidence limit for the empirical mode decomposition and hilbert spectral analysis. P Roy Soc Lond A Mat, 459(2037), 2317-2345.

Puhales, F. S., Demarco, G., Martins, L. G. N., Acevedo, O. C., Degrazia, G. A., Welter, G. S., Costa, F. D., Fisch, G. F., Avelar, A. C. (2015). Estimates of turbulent kinetic energy dissipation rate for a stratified flow in a wind tunnel. Physica A: Statistical Mechanics and its Applications, 431, 175-187.

Wittwer, A. R., y Alvarez, G. M. A., Demarco, G., Martins, L. G. N., Puhales, F. S., Acevedo, O. C., Degrazia, G. A., Bodmann, B., Loredo-Souza, A. M. (2016). Employing wind tunnel data to evaluate a turbulent spectral model. American Journal of Environmental Engineering, 6(4A), 156-159.

Luís Gustavo Nogueira Martins

Universidade Federal de Santa Maria E-mail:1gnm.sm@gmail.com

Giuliano Demarco

Universidade Federal de Santa Maria E-mail: giulianofisico@gmail.com

Franciano Scremin Puhales

Universidade Federal de Santa Maria fpuhales@gmail.com

Gervásio Annes Degrazia

Universidade Federal de Santa Maria E-mail: gervasiodegrazia@gmail.com

Otávio Costa Acevedo

Universidade Federal de Santa Maria, RS, Brasil E-mail: otavio@ufsm.br 